\title{
The Performance of 2.5D Woven Polymeric Matrix Composites under Ballistic Impact Loadings
}

\author{
Weiyi Kong', a , Richard Brooks ${ }^{2}$, Elena Sitnikova ${ }^{2}$, Xiantao Zhao ${ }^{1}$, Shuguang \\ $\mathrm{Li}^{2}$
}

\author{
${ }^{1}$ AECC Commercial Aircraft Engine CO.,LTD., 3998 South Lianhua Road, Minhang District, \\ Shanghai, 201108, China \\ ${ }^{2}$ Polymer Composite Research Group, Department of Mechanical, Material and Manufacturing \\ Engineering, University of Nottingham, University Park, Nottingham, NG7 2RD, United Kindom \\ akongweiyiacae@163.com
}

Keywords: Ballistic impact tests, Polymeric matrix composites, 2.5D woven.

\begin{abstract}
By using a gas gun apparatus, three types of $2.5 \mathrm{D}$ woven composites and one type of laminated composite under ballistic impact loadings were tested under ballistic impact loadings. The trajectory of the projectile was recorded by high speed camera and then analyzed to obtain the impact and residual velocities. The analysis of the experimental data suggests that the GF-2 composite has the highest impact resistance, followed by GF-1, CF-IM7 and QI-IM7. However, if the difference in density is considered, the three types of $2.5 \mathrm{D}$ woven composites have similar efficiency in resisting penetration. The failure and damage modes of the tested plate were visually observed.
\end{abstract}

\section{Introduction}

Polymer matrix composites (PMC), which possess low weight and high static and fatigue strength, have been used to replace metals in aero engines since the early 1970s. When the fan containment casing, one of the largest components in aero engines, is made from PMC rather than mature alloys, its weight can be reduced considerably. The fan containment casing is required to contain the debris generated during fan blade-out events (FBO). Most of the common types of high performance PMC fall into the category of two-dimensional (2D) laminated composites. Due to the lack of reinforcement binding different layers of laminae, the composite laminates are vulnerable to inter-ply delamination, which undermines their impact resistance [1].

3D textile composites [2, 3], such as three-dimensional (3D) woven, 3D braid, stitching and knitting, have been developed to resolve the issue of weak delamination resistance of laminated composites. Among these, 3D weaving process is more suitable for manufacturing large and thick parts [4] like the fan casing. Recently, a special type of 3D woven composites, called 2.5D woven composites [5] was developed. Instead of using the third set of yarns, the through-thickness reinforcement in $2.5 \mathrm{D}$ woven composites is provided by the interlocking warp yarns. Complex near-net-shape $2.5 \mathrm{D}$ woven preforms can be produced on standard industrial weaving looms used for $2 \mathrm{D}$ woven fabrics by introducing minor changes to the weaving loom.

However, research on 2.5D woven PMC is very limited [6-8]. It mainly focuses on developing the numerical tools and methodologies for analysing general 3D textile composites by taking advantage of the simpler architectures of $2.5 \mathrm{D}$ woven textiles. No information regarding the impact performance of $2.5 \mathrm{D}$ woven PMC could be found, both in terms of the modelling work, as well as the experimental investigations. In this paper, the performance of three types of $2.5 \mathrm{D}$ woven PMC under ballistic impact loadings is reported.

\section{Materials}

Flat panels of the 2.5D woven PMC were manufactured from three types of 2.5D textile preform 
panels by applying a vacuum-assisted resin transfer moulding (VARTM) process at the university of Nottingham. Two are made from 75 Tex E-glass fibres and denoted as GF-1 and GF-2. The other one is made from IM7 carbon fibres and denoted as CF-IM7. The resin system used was PRIME ${ }^{\mathrm{TM}}$ 20LV epoxy infusion resin with a slow hardener. All of the preforms have the same basic weaving architecture, namely, 2.5D layer-to-layer angle interlock, whose unit cell is illustrated by Fig. 1. However, there are differences in their geometric parameters, for instance the warp yarn weaving angle and yarn spacing, and also the volume fraction ratio of the warp and weft yarns, as listed in Table 1.

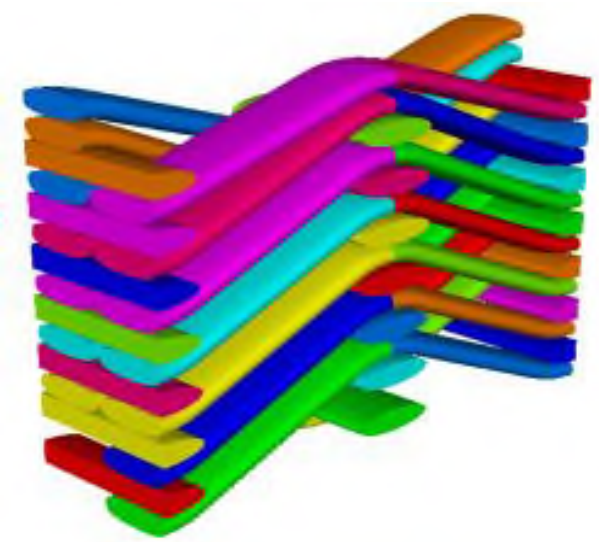

Fig. 1 The unit cell model of 2.5D layer-to-layer angle interlock composites.

Apart from the three types of 2.5D woven composites, a batch of IM7 laminated plates were also tested for comparison purpose. The plates are made from unidirectional HexPly IM7/8552 prepreg. 32 plies of the prepreg were laid up in a quasi-isotropic manner $[0 / \pm 45 / 90]_{s 4}$ and then cured at elevated temperature in an autoclave following the recommended curing schedule. The thickness of the cured plates is $4 \mathrm{~mm}$, similar to those of the $2.5 \mathrm{D}$ woven composites.

Table 1. The volume fractions of yarns and fibres of the three composites, and their unit cell sizes.

\begin{tabular}{ccccc}
\hline Type & $\begin{array}{c}\text { Yarn volume } \\
\text { fraction } \\
\text { (warp: weft) }\end{array}$ & $\begin{array}{c}\text { Overall fibre volume } \\
\text { fraction }\end{array}$ & $\begin{array}{c}\text { Unit cell size } \\
\text { (weft } \times \text { warp) } \\
\text { in mm }\end{array}$ & $\begin{array}{c}\text { Moulded panel } \\
\text { thickness in mm }\end{array}$ \\
\hline GF-1 & $48.99 \%: 13.24 \%$ & $50.95 \% \pm 0.35 \%$ & $9.04 \times 2.62$ & 4.1 \\
GF-2 & $42.62 \%: 23.39 \%$ & $54.00 \% \pm 0.52 \%$ & $8.7 \times 2.62$ & 4.2 \\
CF-IM7 & $41.03 \%: 25.64 \%$ & $55.46 \% \pm 0.34 \%$ & $9.04 \times 2.56$ & 4.2 \\
\hline
\end{tabular}

\section{Methodology}

The performance of GF-1, GF-2 and CF-IM7 under high velocity impact was studied by ballistic impact tests, relevant to the application in aero engine fan casings for containing high velocity debris during FBO. A gas gun apparatus, whose set-up is shown in Fig. 2, is used for this study. 


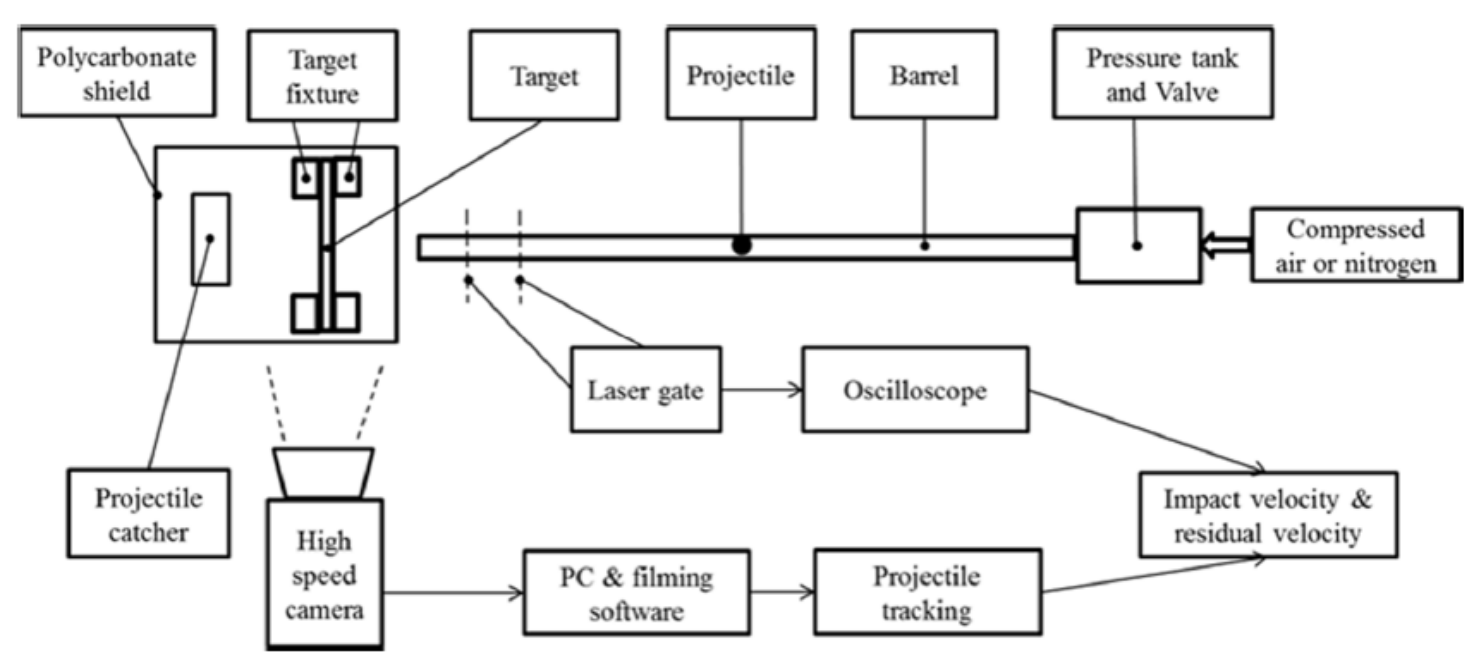

Fig. 2 A schematic of the set-up of ballistic impact test.

The projectiles are steel ball bearings of $12.7 \mathrm{~mm}$ in diameter. They were accelerated in a 3.5-meter-long gun barrel, which was pointing to the centre of the target plates, normal to the surface of the target. The manufactured composite plates were cut into $150 \mathrm{~mm} \times 150 \mathrm{~mm}$ square plates as the targets. Twelve $6 \mathrm{~mm}$ holes were drilled through the target. The target was placed between a fixture plate and a fixture ring, as schematically shown by Fig. 3 (a), to expose a 100-mm-diameter circular area to the impact. The entire target fixture was tightly bolted together by using twelve M6 bolts.
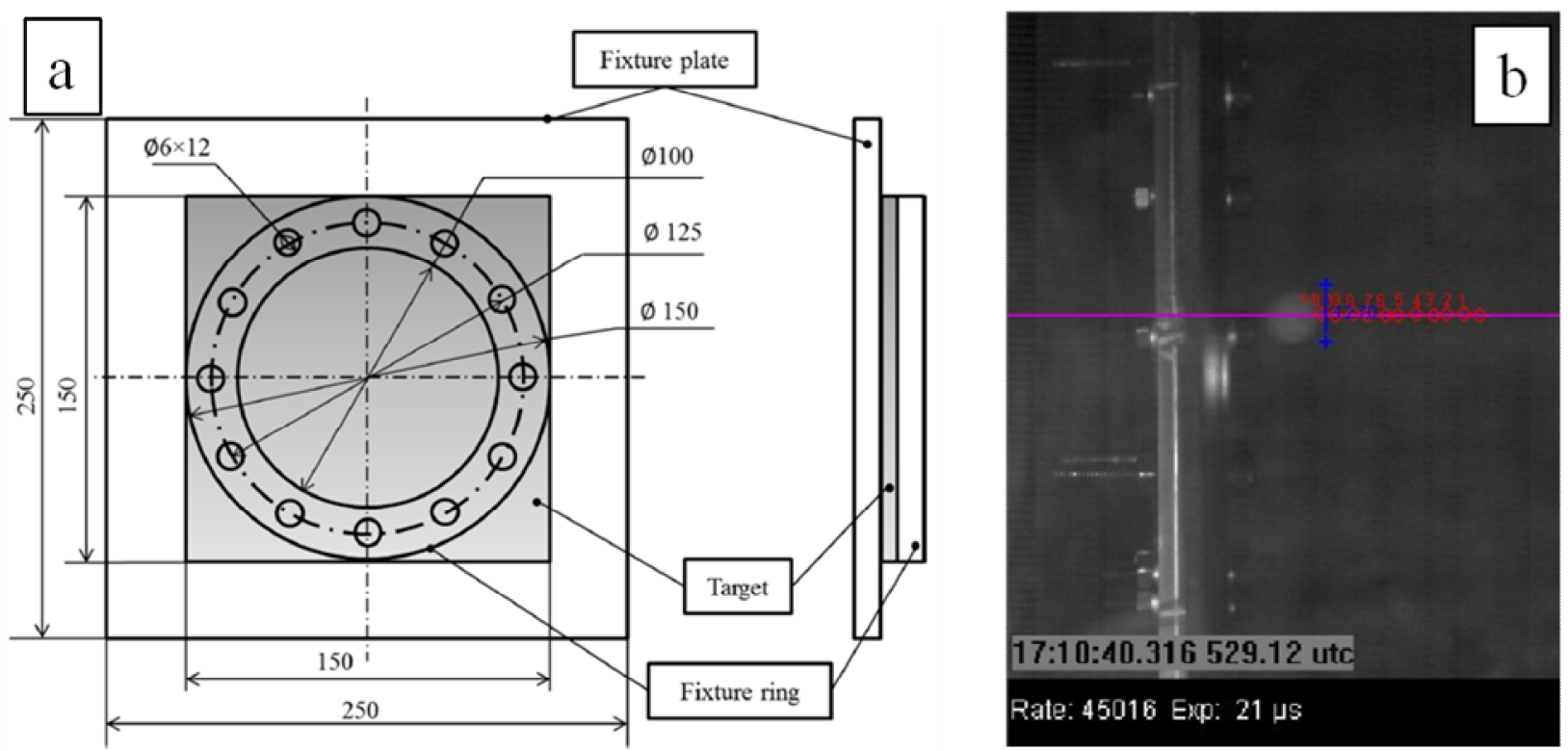

Fig. 3 (a) A schematic of the target with the fixture and their dimensions; (b) One frame of a video showing the impactor before hitting the target.

In the vicinity of the muzzle, two laser gates were placed $10 \mathrm{~cm}$ away from each other to provide approximate but instant measurement of the projectile 80 velocity using an oscilloscope. A Phantom high speed camera along with two Dedocool focused lights was used to film the entire impact process. Each impact test was recorded by the high speed camera as a video file. The impact and residual velocities of the ball were obtained by processing the video file.

Based on the frame rate as recorded in the video files, the time intervals between the frames were determined. As an example, a single frame of a video file is shown in Fig. 3 (b). The dimensions in the videos are calibrated with respect to the known dimensions, for example the diameter of the projectile as shown in blue in Fig. 3 (b). Then, the position of the projectile, as coordinates in each frame, is determined by tracking the point on the leading "edge" of the ball. The horizontal 
coordinate of the projectile is plotted versus time. The velocity was determined by calculating the gradient of the linear-fitting curve of the plot. For all the tests, the data points fit very well with linear curves, indicating constant velocities. The air drag to the projectile is calculated and found to have negligible effect on the projectile velocity.

\section{Test Results and Analysis}

The experimental data are plotted in Fig. 4 as impact velocities $V_{\mathrm{i}}$ and residual velocities $V_{\mathrm{r}}$. The negative residual velocities indicate those cases where the projectile rebounded. A commonly used indicator of impact resistance is the limit velocity $V_{50}$, at which the projectile penetrates the target at least $50 \%$ of the time. To save material and effort in determining $V_{50}$, interpolation or extrapolation is often applied as is the case in this work. Specifically, the Jonas-Lambert equation [9] is commonly used for this purpose. It is expressed as

$$
V_{1}^{p}=\beta^{p}\left(V_{\mathrm{i}}^{p}-V_{\mathrm{so}}^{p}\right)
$$

where $\beta$ and $p$ are the parameters, and $V_{50}$ is also a parameter of the fitting function. It should be noted that this expression is not applicable for the rebounding cases. The nonlinear curve fitting was applied to determine $V_{50}, \beta$ and $p$ in the Curve Fitting Toolbox of MatLab 2013.

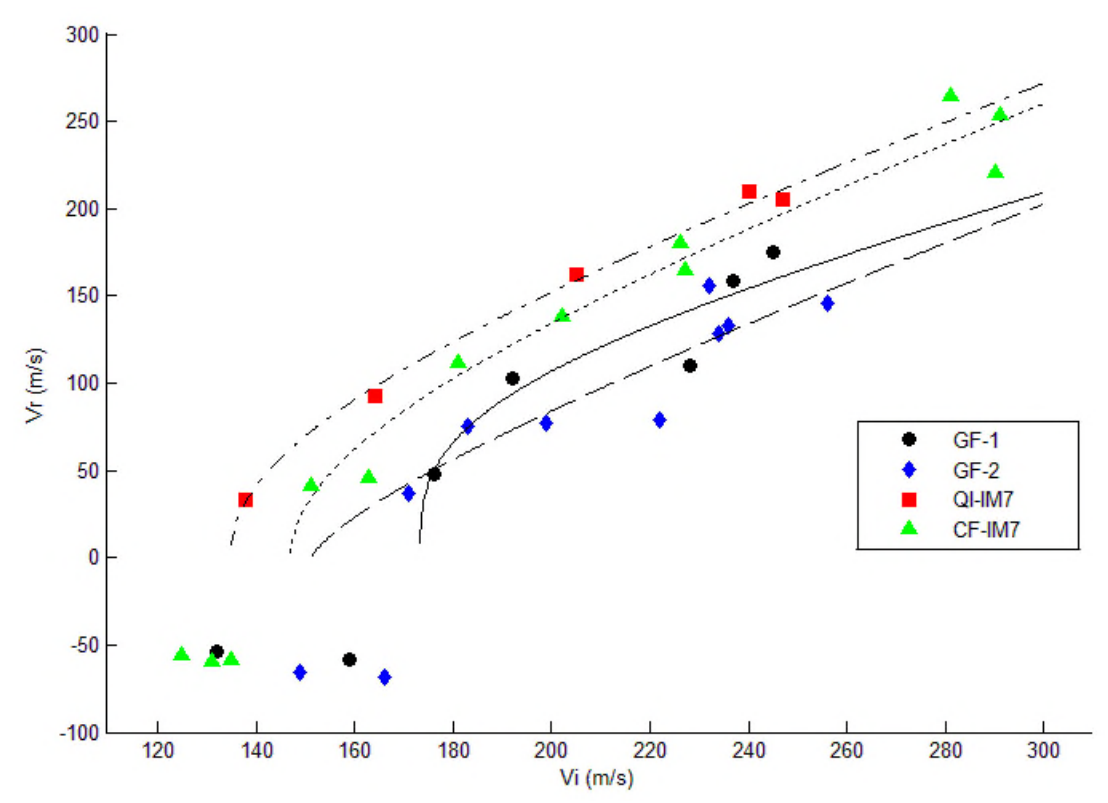

Fig. 4 Ballistic impact test data fitted with Jonas-Lambert equation for four composites.

The fitting parameters and their standard errors are given in Fig. 5. It can be seen that some errors are very large. Prediction bands were also computed as shown in Fig. 5. They enclose an area which a new test should be in with $95 \%$ probability. The wider are the prediction bands, the larger are the errors in the fitting parameters. As can be seen, the limit velocity can be estimated reasonably accurately based on the ballistic impact data on CF-IM7 textile composite as well as QI laminates while the fit is generally unreliable for GF-1 and GF-2. In particular, the prediction bands for GF-1 are the widest because of the standard error of the exponent $p$, which was also the largest. The uncertainty in definition of a fitting curve in these two cases arises primarily from the scatter of the experimental data based on which fitting function was calculated. 


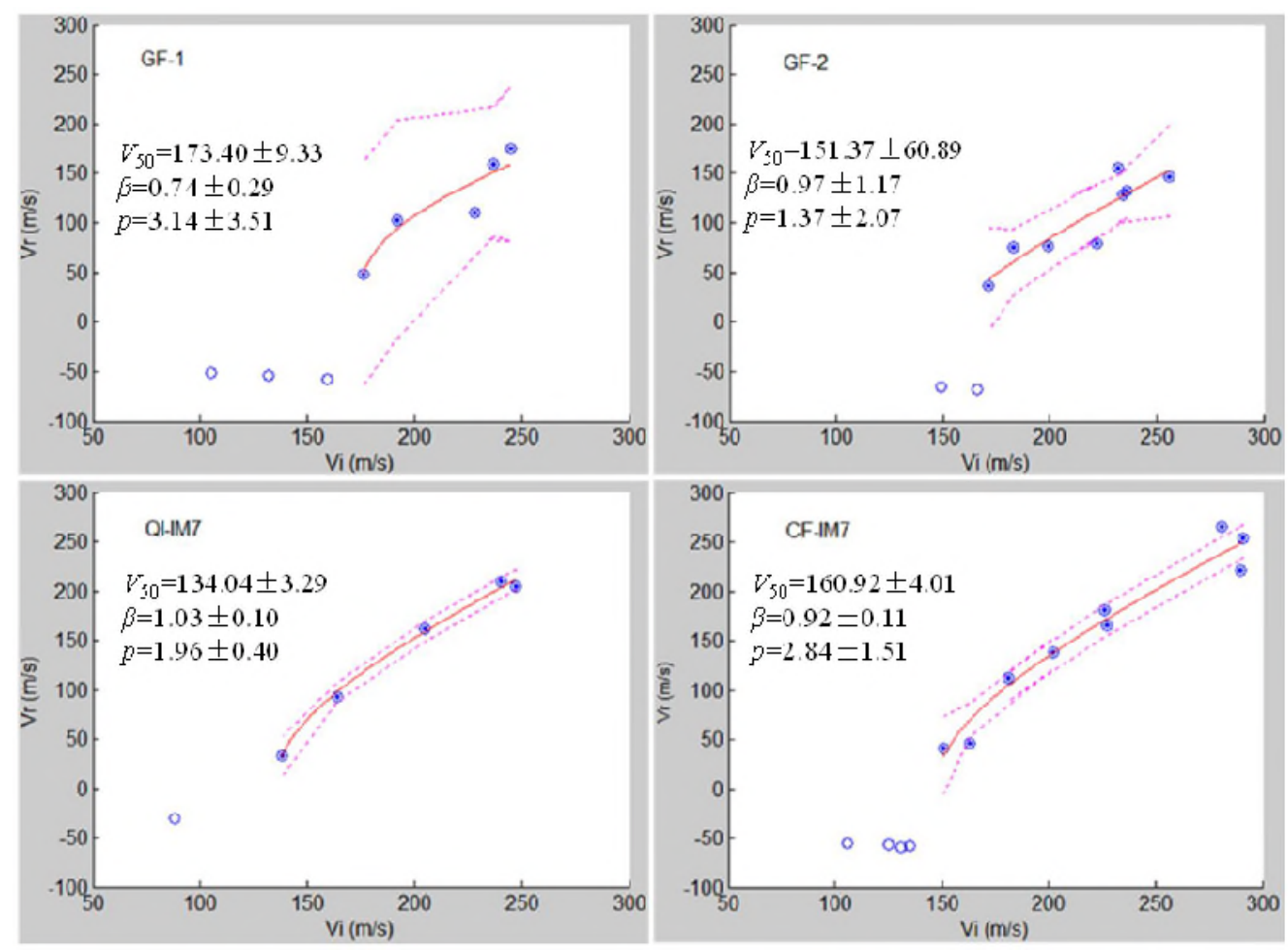

Fig. 5 Prediction bands of the fitting functions of ballistic impact test data.

Despite the poor fitting, it is possible to make a rough assessment on the impact performance of GF-1 and GF-2 based on the experimental data available. Comparing the experimental data in Fig. 4, it is easy to see that the residual velocities for these two materials are lower than those for CF-IM7 and QI laminate, which indicates that they can dissipate a larger amount of energy during the impact, hence should have a higher impact resistance. As reference values for the critical velocity, the $V_{50}=$ $173 \mathrm{~m} / \mathrm{s}$, as was determined by fitting the experimental data, was assumed for GF-1 composite. For GF-2, although the estimated value for $V_{50}$ has a very large standard error, it can be seen in Fig. 4, most of the GF-2 points are located to the right of the GF-1 ones. This indicates that GF-2 possibly requires a larger impact velocity than GF-1 to achieve the same level of residual velocity. In other words, the ballistic limit of GF-2 is higher than GF-1. Hence in this study, $V_{50}=180 \mathrm{~m} / \mathrm{s}$, was chosen as a reference value for GF-2. The ballistic limits for all four composites are summarised in Table 2.

The limit velocities, as well as the Lambert extrapolating curves, lead to the same conclusions about the impact resistance. The QI-IM7 laminates has the lowest resistance to impact. If it is used as a baseline, when the IM7 fibres are woven into the $2.5 \mathrm{D}$ reinforcements, the limit velocity is increased by about $20 \%$ and its corresponding energy is increased by $44 \%$.

The resistance of the glass fibre composites seems to be higher than the carbon fibre composites. However, glass fibres are known to have larger density than carbon fibres. The plates in this study have similar thickness, hence the glass plates are heavier. If the above energies are normalised to the densities of the plates (as in Table 2), the glass composites GF-1 and GF-2 show almost the same impact resistant as the carbon composite CF-IM7.

Table 2. Ballistic limit $V_{50}$ of the four composites.

\begin{tabular}{lcccc}
\hline & GF-1 & GF-2 & CF-IM7 & QI-IM7 \\
\hline$V_{50}(\mathrm{~m} / \mathrm{s})$ & 173 & $180^{*}$ & 161 & 134 \\
Normalised energy $^{1}$ & $169 \%$ & $180 \%$ & $144 \%$ & $100 \%$ \\
Normalised energy $^{2}$ & $132 \%$ & $141 \%$ & $134 \%$ & $100 \%$ \\
\hline
\end{tabular}

1. The kinetic energy at the ballistic limit is normalised to the one of QI-IM7.

2. The normalised energy is further normalised to the densities of the plates 


\section{Damage and Failure after the Ballistic Impact}

The internal damage due to impact is visually observed for the semi-transparent glass composite plates. Visual observation is not applicable for opaque CF-IM7 plates. As an example, Fig. 6 shows the back face of a GF-2 plate penetrated at $256 \mathrm{~m} / \mathrm{s}$, where four types of failure and damage can be observed, in addition to the obvious modes of crushing and fibre breakage at the impact centre. The red lines were drawn prior to impact to find the centre of the plate. They are irrelevant to the following observation.

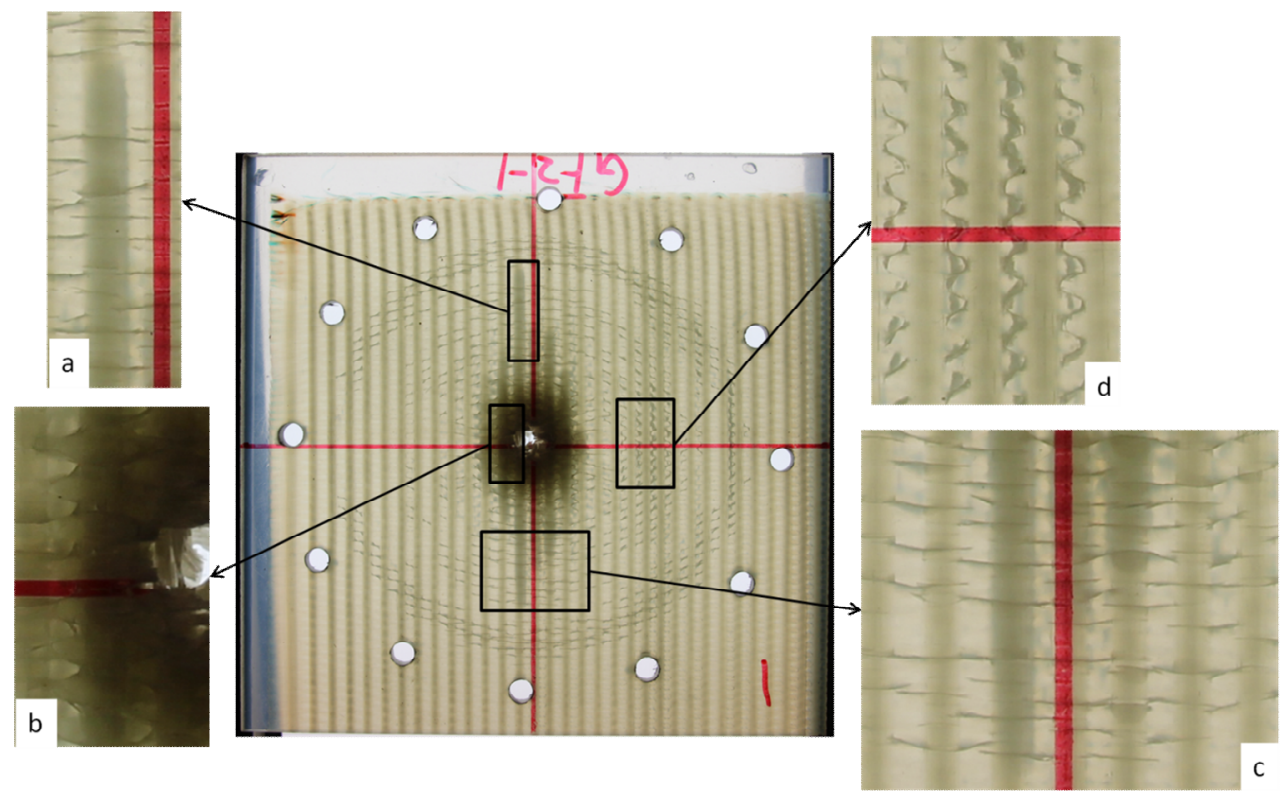

Fig. 6 A penetrated GF-2 plate with four damage modes.

The damaged weft yarns, as shown by Fig. 6 (a), are more opaque than the intact ones. They extend far from the impact point and nearly to the circular periphery in this particular case, due to the stretching by the projectile during the impact. The warp yarn damage in Fig. 6 (b) is mainly localised to the impact centre, as crimped warp yarns do not transfer much of the stretching loading.

Two types of matrix damage took place at various locations of the plate. The damage in Fig. 6 (c) are matrix cracks normal to the weft yarns. Wavy patterns of debonding in resin rich regions around the superficial layers of warp yarns, as shown by Fig. 6 (d), are found as the other matrix damage mode. These two damage modes spread over the target, even for the impact in Fig. 6, which tends to be a localised event as the impact velocity is well above the ballistic limit. A circular region of cracks of both types is also found around the periphery.

These damage modes are commonly observed in all the tested glass fibre plates, visible from both front and back faces of the impacted plates, although the extents differ. As examples, Fig. 7 shows three plates of GF-1 tested at $159 \mathrm{~m} / \mathrm{s}, 176 \mathrm{~m} / \mathrm{s}$ and $237 \mathrm{~m} / \mathrm{s}$, along with another three of GF-2 impacted at $149 \mathrm{~m} / \mathrm{s}, 199 \mathrm{~m} / \mathrm{s}$ and $256 \mathrm{~m} / \mathrm{s}$. Note that the limit velocities are $173 \mathrm{~m} / \mathrm{s}$ and 180 $\mathrm{m} / \mathrm{s}$ for GF-1 and GF-2, respectively. It can be seen that the damage of weft yarns in the centre of the panel appears to be more extended in GF-2 than in GF-1. This indicates the denser weft yarns of GF-2 took more part in resisting penetration than those of GF-1. 

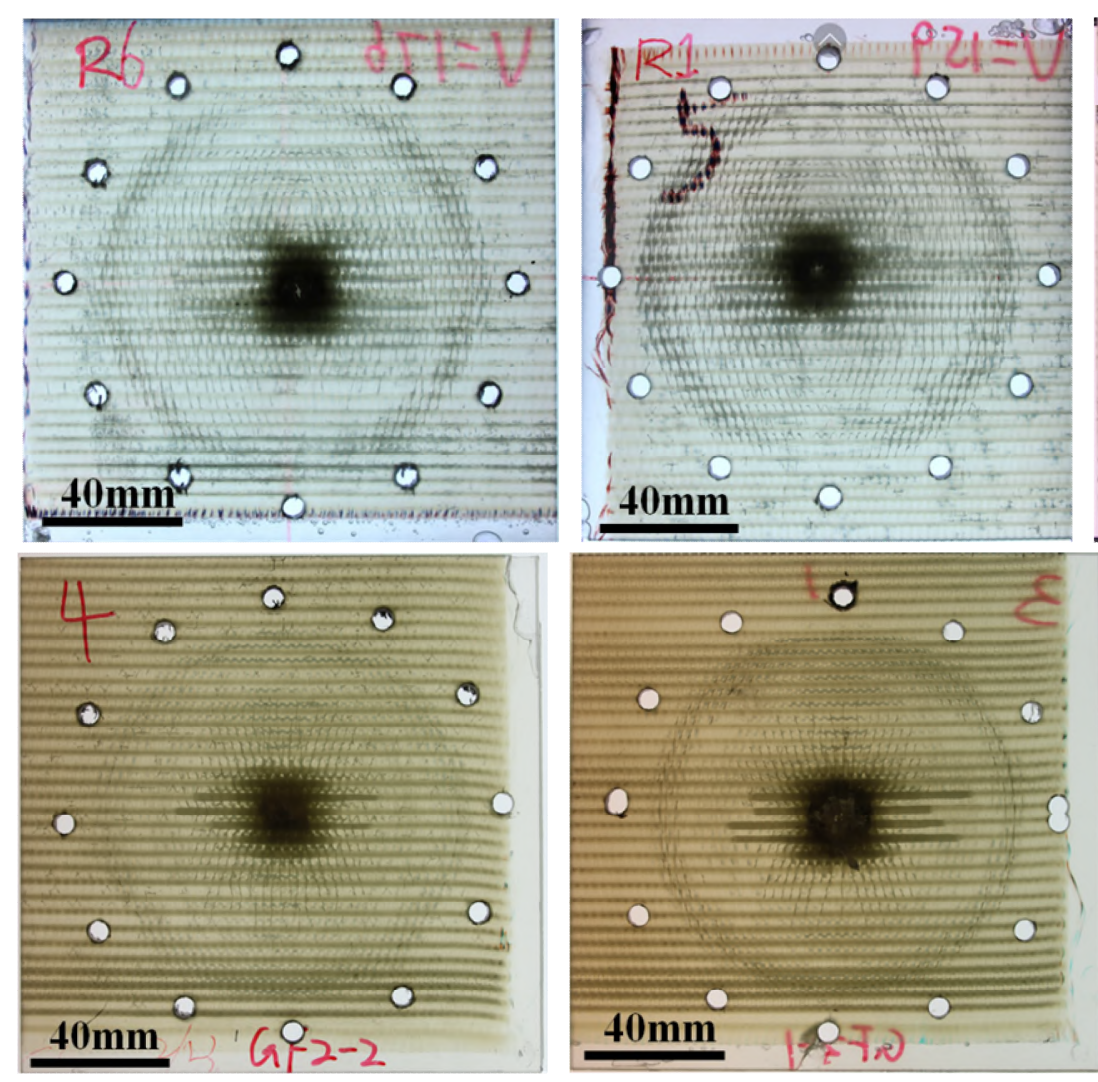
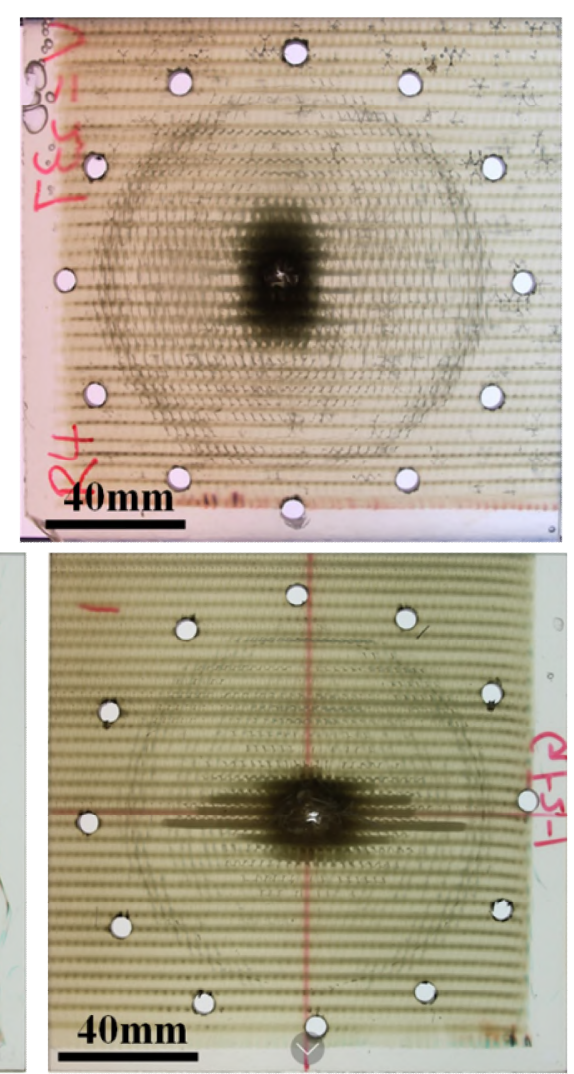

Fig. 7 The plates of GF-1 impacted at $159 \mathrm{~m} / \mathrm{s}, 176 \mathrm{~m} / \mathrm{s}$ and $237 \mathrm{~m} / \mathrm{s}$ on the first row; The plates of GF-2 impacted at $149 \mathrm{~m} / \mathrm{s}, 199 \mathrm{~m} / \mathrm{s}$ and $256 \mathrm{~m} / \mathrm{s}$ on the second row. All is viewed from the back face of impact.

\section{Conclusion}

Three types of 2.5D woven composites and one type of laminated composite were tested by performing ballistic impact tests. The results indicate the $2.5 \mathrm{D}$ woven composites are more resistant to penetration than the laminated composites. When the difference in density is considered, the three types of $2.5 \mathrm{D}$ woven composites have similar efficiency in resisting penetration. In addition to the matrix damage which spread all over the plate, localised warp yarn damage and extended weft yarn damage were observed by visual inspecting the tested glass-fibre plates. The denser weft yarns of GF-2 took more part in resisting penetration and the observed impact resistance is better than GF-1.

\section{Acknowledgement}

The authors would like to acknowledge the financial support provided by Science and Technology Commission of Minhang District, Shanghai City, China, through Grant 201501-MHC101-007.

\section{References}

[1] S. Abrate, Impact on Laminated Composite Materials, Appl. Mech. Rev. 44(4) (1991) 155-190.

[2] A. Miravete, 3-D textile reinforcements in composite materials, 1999: Woodhead Publishing.

[3] L. Tong, A. P. Mouritz, M. Bannister, 3D fibre reinforced polymer composites, 2002: Elsevier.

[4] A. P. Mouritz, M. K. Bannister, P. J. Falzon, K. H. Leong, Review of applications for advanced three-dimensional fibre textile composites, Compos. Part A: Appl. Sci. Manuf. 30(12) (1999) 1445-1461.

[5] A. Dalmaz, D. Ducret, R. El Guerjouma, P. Reynaud, P. Franciosi, D. Rouby, G. Fantozzi, J. C. 
Baboux, Elastic moduli of a $2.5 \mathrm{D} \mathrm{Cf} / \mathrm{SiC}$ composite: experimental and theoretical estimates, Compos. Sci. Technol. 60(6) (2000) 913-925.

[6] S. Nehme, A. Hallal, F. Fardoun, R. Younes, B. Hagege, Z. Aboura, M. Benzeggagh, F. H. Chehade, Numerical/analytical methods to evaluate the mechanical behavior of interlock composites, J. Compos. Mater. 45(16) (2011) 1699-1716.

[7] Z. Lu, Y. Zhou, Z. Yang, Q. Liu, Multi-scale finite element analysis of 2.5D woven fabric composites under on-axis and off-axis tension, Comput. Mater. Sci. 79 (2013) 485-494.

[8] A. Hallal, R. Younes, F. Fardoun, S. Nehme, Improved analytical model to predict the effective elastic properties of 2.5D interlock woven fabrics composite, Compos. Struct. 94(10) (2012) 3009-3028.

[9] J. Lambert, G. Jonas, Towards standardization in terminal ballistics testing: velocity representation, 1976, DTIC Document. 